\title{
NULL CONTROLLABILITY OF A NONLINEAR POPULATION DYNAMICS PROBLEM
}

\author{
OUMAR TRAORE
}

Received 23 November 2005; Revised 8 August 2006; Accepted 11 October 2006

We establish a null controllability result for a nonlinear population dynamics model. In our model, the birth term is nonlocal and describes the recruitment process in newborn individuals population. Using a derivation of Leray-Schauder fixed point theorem and Carleman inequality for the adjoint system, we show that for all given initial density, there exists an internal control acting on a small open set of the domain and leading the population to extinction.

Copyright (c) 2006 Hindawi Publishing Corporation. All rights reserved.

\section{Introduction}

For a given positive real function $F$, we consider in this paper the following nonlinear population dynamics model:

$$
\begin{gathered}
\frac{\partial y}{\partial t}+\frac{\partial y}{\partial a}-\Delta y+\mu y=v 1_{\omega} \quad \text { in }(0, T) \times(0, A) \times \Omega, \\
y(t, a, \sigma)=0 \quad \text { on }(0, T) \times(0, A) \times \partial \Omega, \\
y(0, a, x)=y_{0}(a, x) \quad \text { in }(0, T) \times(0, A) \times \Omega, \\
y(t, 0, x)=F\left(\int_{0}^{A} \beta(t, a, x) y(t, a, x) d a\right) \quad \text { on }(0, T) \times \Omega,
\end{gathered}
$$

where $\Omega$ is a bounded open subset of $\mathbb{R}^{N}, N \geq 1$ with a smooth boundary $\partial \Omega, \sigma \in \partial \Omega$, $T$ is a positive real and $\omega$ an open subset such that $\bar{\omega} \subset \Omega$. Here $y(t, a, x)$ is the distribution of individuals of age $a$ at time $t$ and location $x \in \Omega, 1_{\omega}$ is the characteristic function of $\omega, A$ is the maximal live expectancy, $\Delta$ the Laplacian with respect to the spatial variable, $\beta(t, a, x)$ and $\mu(t, a, x)$ denote, respectively, the natural fertility and the natural death rate of individuals of age $a$ at time $t$ and location $x$. Thus, the formula $\int_{0}^{A} \beta(t, a, x) y(t, a, x) d a$ denotes the distribution of newborn individuals at time $t$ and location $x$. In an oviparus species it denotes the total eggs at time $t$ and position $x$. Therefore, the quantity $F\left(\int_{0}^{A} \beta(t, a, x) y(t, a, x) d a\right)$ is the distribution of eggs that hatches at time $t$ and position $x$. 
System (1.1) describes the evolution of an internal controlled age and space structured population under inhospitable boundary conditions in the case that the flux of individuals has the form $-\nabla y(t, a, x)$.

The purpose of this paper is to prove a null controllability result for (1.1) at any time $T$. This means more precisely that there exists a control $v \in L^{2}((0, T) \times(0, A) \times \omega)$ such that the associated solution of (1.1) verifies

$$
y(T, a, x)=0 \quad \text { a.e. in }(0, A) \times \Omega .
$$

In our knowledge the first controllability result for an age and space structured population dynamics model was established by Ainseba and Langlais in [4]: they proved that a set of profiles is approximately reachable. In [2] a local exact controllability result was proved for a linear population dynamics. More precisely, in [2] the authors proved that if the initial distribution is small enough, one can find a control that leads the population to extinction. The method used there is different from ours. In fact in [2] the adjoint system was taken as a collection of parabolic equations along characteristic lines. This allowed the authors to use Carleman inequality for parabolic equation. Ainseba and Iannelli in [3] proved a null controllability result for a nonlinear population dynamics model. In [3] the natural rates depend on the total population $P=\int_{0}^{A} y(t, a, x) d a$. The method in [3] used Kakutani fixed point theorem. Therefore, crucial assumptions were made: first, the natural rates were supposed to be globally Lipschitz with respect to the variable $P$, secondly in order to perform key estimates, the death rate $\mu$ verified the following growth condition: $0 \leq \mu \exp \left(\int_{0}^{a} \mu(s) d s\right) \leq \zeta$ where $\zeta$ is a positive constant.

In the case we study here, the above results cannot be applied. Indeed, since the birth process is not globally Lipschitz with respect to the variable $P$ and, without the previous growth condition on $\mu$ one cannot use the method of [3]. On the other hand, the nonlinearity excludes the use of the result of [2]. In what follows, using a Carleman inequality for an adjoint system we establish a null controllability result for the nonlinear population dynamics models stated in (1.1) when the initial distribution is in $L^{2}((0, A) \times \Omega)$. Roughly, in our method we first study a null controllability result for a population in which the birth process is given by a fixed function. Afterwards, we prove the null controllability result for the system (1.1) by means of a derivation of Leray-Shauder theorem.

The remainder of this paper is as follows: in Section 2, we state assumptions and we provide the main result. In Section 3 we study a null controllability result for some associated model. Section 4 is devoted to the proof of the main result.

\section{Assumptions and main result}

For the sequel we assume that the following assumptions hold:

$$
H_{1}\left\{\begin{array}{l}
\mu(t, a, x)=\mu_{0}(a)+\mu_{1}(t, a, x) \quad \text { a.e. in }(0, T) \times(0, A) \times \Omega, \\
\mu \geq 0 \quad \text { a.e. in }(0, T) \times(0, A) \times \Omega, \\
\mu_{1} \in L^{\infty}((0, T) \times(0, A) \times \Omega) ; \quad \mu_{1}(t, a, x) \geq 0 \quad \text { a.e. in }(0, T) \times(0, A) \times \Omega, \\
\mu_{0} \in L_{\mathrm{loc}}^{1}(0, A), \quad \lim _{a \rightarrow A} \int_{0}^{a} \mu_{0}(s) d s=+\infty,
\end{array}\right.
$$




$$
H_{2}\left\{\begin{array}{l}
\beta \in C^{2}([0, T] \times[0, A] \times \bar{\Omega}), \\
\beta(t, a, x) \geq 0 \quad \text { in }[0, T] \times[0, A] \times \bar{\Omega}, \\
\exists 0<a_{0}<a_{1}<A \quad \text { such that } \beta(t, a, x)=0 \text { in }[0, T] \times\left(\left(0, a_{0}\right) \cup\left(a_{1}, A\right) \times \Omega\right) .
\end{array}\right.
$$

$H_{3} F$ defined on $\mathbb{R}$ is a positive continuous function and there exist positive constants $C_{0}$ and $C_{1}$ such that $F(t) \leq C_{0}+C_{1}|t|$, for all $t \in \mathbb{R}$.

Remark 2.1. Since $\mu$ and $\beta$ are natural rates, the second assumptions of $H_{1}$ and $H_{2}$ are natural. The third assumption of $\mathrm{H}_{2}$ is also natural, since it means that older and younger individuals are not fertile. The fourth assumption in $H_{1}$ is also a standard one, it means that all individual dies before the age $A$. In [3] the model did not take explicitly into account the death of newborns. Indeed the birth process there has the form $y(t, 0, x)=\int_{0}^{A} \beta(t, a, x, P(t, x)) y(t, a, x) d a$ where $P(t, x)=\int_{0}^{A} y(t, a, x) d a$. We present here a quite different model. In fact our model addresses both supply and death of newborns. Moreover in the case $F(t)=k t$ with $k$ a fixed positive constant, one obtains from (1.1) a linear population dynamics problem.

Assume now that the function $F$ is a globally Lipschitz one and verifies $F(0)=0$. Then, one can rewrite $F$ as $F(t)=t \Phi(t)$ for a.e. $t \in \mathbb{R}$. Therefore, the fourth equation of (1.1) becomes $y(t, 0, x)=\int_{0}^{A} \beta y d a \Phi\left(\int_{0}^{A} \beta y d a\right)$. Hence, one obtains the system considered with Neumann boundary conditions in $[8,10]$ where existence of solution was studied.

From now we set $Q=(0, T) \times(0, A) \times \Omega ; q=(0, T) \times(0, A) \times \omega ; Q_{A}=(0, A) \times \Omega$; $Q_{T}=(0, T) \times \Omega ; \Sigma=(0, T) \times(0, A) \times \partial \Omega$ and $C_{\beta}=\|\beta\|_{C^{2}(\bar{Q})}$.

For $\alpha \geq 0$ we set $S_{\alpha}(t, a)=\exp \left(-\alpha t+\int_{0}^{a} \mu_{0}(s) d s\right), X_{\alpha}=\left\{z \in L^{2}\left(Q_{A}\right) ; S_{\alpha}(t, a) z \in L^{2}\left(Q_{A}\right)\right\}$, and $Y_{\alpha}=\left\{v \in L^{2}(q) ; S_{\alpha}(t, a) v \in L^{2}(q)\right\}$. It is obvious that $\alpha_{1} \geq \alpha_{2}$ implies $X_{\alpha_{1}} \subset X_{\alpha_{2}}$ and $Y_{\alpha_{1}} \subset Y_{\alpha_{2}}$.

In the sequel, $\nu$ will denote the unit outward normal vector to $\partial \Omega$ and $C(\Omega, T, A, \ldots)$ will denote positive constant that depends only on $\Omega, T, A, \ldots$

We are now ready to state the main result of this paper.

Theorem 2.2. For any $\gamma>0$ assumed to be small enough, there exists a control $v \in Y_{0}$ such that the associated solution of (1.1) satisfies

$$
y(T, a, x)=0 \quad \text { a.e. in }(\gamma, A) \times \Omega
$$

for all $y_{0} \in X_{0}$.

Remark 2.3. In the proof, it will appear clearly that such a control depends essentially on $\gamma$.

Let us denote by $\lambda_{0}$ a positive constant which will be fixed later. We make the following standard changes: $\hat{y}=S_{\lambda_{0}}(t, a) y, \hat{v}=S_{\lambda_{0}}(t, a) v, \widehat{\beta}=S_{\lambda_{0}}^{-1}(0, a) \beta$ and $\widehat{y_{0}}=S_{\lambda_{0}}(t, a) y_{0}$. Then 


\section{Controllability of a nonlinear population model}

it follows that $\hat{y}$ solves the following system:

$$
\begin{gathered}
\frac{\partial \hat{y}}{\partial t}+\frac{\partial \hat{y}}{\partial a}-\Delta \hat{y}+\left(\mu_{1}+\lambda_{0}\right) \hat{y}=\hat{v} 1_{\omega} \quad \text { in } Q, \\
\hat{y}(t, a, \sigma)=0 \quad \text { on } \Sigma, \\
\hat{y}(0, a, x)=\hat{y}_{0}(a, x) \quad \text { in } Q_{A}, \\
\hat{y}(t, 0, x)=e^{-\lambda_{0} t} F\left(e^{\lambda_{0} t} \int_{0}^{A} \hat{\beta}(t, a, x) \hat{y}(t, a, x) d a\right) \quad \text { in } Q_{T} .
\end{gathered}
$$

The null controllability problem of Theorem 2.2 is now reduced to find $\hat{v}$ in $L^{2}(q)$ such that $\hat{y}$ verifies (2.2). In fact after the previous change we obtain a system involving bounded coefficients and this allows one to establish a global Carleman inequality. In the sequel for the sake of simplicity, we will consider only the previous system without hats and in addition we will write $\mu$ instead of $\mu_{1}+\lambda_{0}$.

\section{Null controllability for some linearized model}

3.1. An observability inequality result. We recall here that there exists a function $\Psi \in$ $C^{2}(\bar{\Omega})$ such that $\Psi(x)=0$, for all $x \in \partial \Omega ; \Psi(x)>0$, for all $x \in \Omega$ and $\nabla \Psi(x) \neq 0$, for all $x \in \overline{\Omega-\widetilde{\omega}}$ where $\tilde{\omega}$ is an open set such that $\widetilde{\widetilde{\omega}} \subset \omega \subset \Omega$. (See [6] for the existence of $\Psi$.)

Let us consider the following system:

$$
\begin{gathered}
-\frac{\partial w}{\partial t}-\frac{\partial w}{\partial a}-\Delta w+\mu w=f \quad \text { in } Q \\
w(t, a, \sigma)=0 \quad \text { on } \Sigma \\
w(T, a, x)=g(a, x) \quad \text { in } Q_{A}, \\
w(t, A, x)=0 \quad \text { in } Q_{T} .
\end{gathered}
$$

Setting for all positive real $\lambda, \eta(t, a, x)=\left(e^{2 \lambda\|\Psi\|_{\infty}}-e^{\lambda \Psi(x)}\right) / a t(T-t)$ and $\varphi(t, a, x)=e^{\lambda \Psi(x)} /$ at $(T-t)$ one can prove easily by adapting the method of [6] or [9] the following.

Proposition 3.1. There exist positive constants $s_{1} \geq 1$ and $\lambda_{1} \geq 1$ and there exists a positive constant $C$ such that for all $s \geq s_{1}, \lambda \geq \lambda_{1}$, and for all solution of (3.1), the following inequality holds:

$$
\int_{Q} e^{-2 s \eta} s^{3} \varphi^{3} \lambda^{4} w^{2} d t d a d x \leq C\left(\int_{Q} e^{-2 s \eta} f^{2} d t d a d x+\int_{q} e^{-2 s \eta} s^{3} \varphi^{3} \lambda^{4} w^{2} d t d a d x\right) .
$$

Remark 3.2. The proof of Proposition 3.1 is absolutely similar to those of global Carleman inequality for the linear heat equation proposed in [9] or in [6]. Roughly, for the proof of (3.2), one makes the change of variable: $u=e^{-s \eta} w$ in order to get from the definition of $\eta$ the following:

$$
u(0, a, x)=u(T, a, x)=u(t, 0, x)=0 .
$$

Subsequently, one derives estimates on $u$ and after return to $w$. We will prove first (3.2) for a function $w \in C^{2}(\bar{Q})$ and after the result for $w \in L^{2}(Q)$ will follow by density arguments. 
Proof of Proposition 3.1. We suppose that the function $w \in C^{2}(\bar{Q})$ and verifies (3.1) and we make the following change of variables $u=e^{-s \eta} w$. Then immediately it follows by using the definition of $\eta$ and (3.1) that

$$
\begin{aligned}
& u(0, a, x)=u(T, a, x)=0 \quad \text { in }(0, A) \times \Omega, \\
& u(t, 0, x)=u(t, A, x)=0 \quad \text { in }(0, T) \times \Omega, \\
& u(t, a, \sigma)=0 \quad \text { in }(0, T) \times(0, A) \times \partial \Omega .
\end{aligned}
$$

Notice that

$$
\begin{gathered}
\nabla \eta=-\lambda \varphi \nabla \Psi \\
\nabla \varphi=\lambda \varphi \nabla \Psi .
\end{gathered}
$$

Using once again the definitions of $\eta$ and $\varphi$, we deduce that there exist positive constants denoted by $C$ such that $|\partial \eta / \partial a| \leq C \varphi^{2},|\partial \eta / \partial t| \leq C \varphi^{2},\left|\partial^{2} \eta / \partial a \partial t\right| \leq C \varphi^{3}$, and $\left|\partial \eta / \partial a^{2}\right| \leq$ $C \varphi^{3}$.

Similarly we get

$$
\left|\frac{\partial \varphi}{\partial a}\right| \leq C \varphi^{2}, \quad\left|\frac{\partial \varphi}{\partial t}\right| \leq C \varphi^{2}, \quad\left|\frac{\partial^{2} \varphi}{\partial a \partial t}\right| \leq C \varphi^{3}, \quad\left|\frac{\partial \varphi}{\partial a^{2}}\right| \leq C \varphi^{3} .
$$

We have

$$
\frac{\partial u}{\partial t}+\frac{\partial u}{\partial a}=-s\left(\frac{\partial \eta}{\partial t}+\frac{\partial \eta}{\partial a}\right) u+e^{-s \eta}\left(\frac{\partial w}{\partial t}+\frac{\partial w}{\partial a}\right) .
$$

From (3.7) and (3.8) we get

$$
\Delta u=s \lambda \Delta \Psi \varphi u+s \lambda^{2}|\nabla \Psi|^{2} \varphi u-s^{2} \lambda^{2}|\nabla \Psi|^{2} \varphi^{2} u+2 s \lambda \varphi \nabla \Psi \cdot \nabla u+e^{-s \eta} \Delta w .
$$

Therefore

$$
\begin{aligned}
& -\left(\frac{\partial u}{\partial t}+\frac{\partial u}{\partial a}\right)-\Delta u+\mu u \\
& \quad=e^{-s \eta} f-s \lambda^{2} u \varphi|\nabla \Psi|^{2}-2 s \lambda \varphi \nabla \Psi \cdot \nabla u+s^{2} \lambda^{2} \varphi^{2}|\nabla \Psi|^{2} u+s\left(\frac{\partial \eta}{\partial t}+\frac{\partial \eta}{\partial a}\right) u-s \lambda \varphi u \Delta \Psi
\end{aligned}
$$

This equation can be rewritten as

$$
P_{1} u+P_{2} u=g_{s}
$$

where

$$
\begin{gathered}
P_{1} u=-\frac{\partial u}{\partial t}-\frac{\partial u}{\partial a}+2 s \lambda \varphi \nabla \Psi \cdot \nabla u+2 s \lambda^{2} u \varphi|\nabla \Psi|^{2}, \\
P_{2} u=-\Delta u-s\left(\frac{\partial \eta}{\partial t}+\frac{\partial \eta}{\partial a}\right) u-s^{2} \lambda^{2} \varphi^{2}|\nabla \Psi|^{2} u, \\
g_{s}=e^{-s \eta} f+s \lambda^{2} u \varphi|\nabla \Psi|^{2}-\mu u-s \lambda u \varphi \Delta \Psi .
\end{gathered}
$$




\section{Controllability of a nonlinear population model}

Taking the square of (3.13) and integrating the result over $Q$ yield

$$
\int_{Q}\left|P_{1} u\right|^{2} d t d a d x+\int_{Q}\left|P_{2} u\right|^{2} d t d a d x+2 \int_{Q} P_{2} u P_{1} u d t d a d x=\int_{Q} g_{s}^{2} d t d a d x .
$$

Let us compute $K=\int_{Q} P_{2} u P_{1} u d t d a d x$.

We obtain

$$
\begin{aligned}
K= & \int_{Q}\left(-\frac{\partial u}{\partial t}-\frac{\partial u}{\partial a}+2 s \lambda \varphi \nabla \Psi \cdot \nabla u+2 s \lambda^{2} u \varphi|\nabla \Psi|^{2}\right)\left(-\Delta u-s\left(\frac{\partial \eta}{\partial a}+\frac{\partial \eta}{\partial t}\right) u\right) d t d a d x \\
& -\int_{Q}\left(-\frac{\partial u}{\partial t}-\frac{\partial u}{\partial a}+2 s \lambda \varphi \nabla \Psi \cdot \nabla u+2 s \lambda^{2} u \varphi|\nabla \Psi|^{2}\right) s^{2} \lambda^{2} \varphi^{2}|\nabla \Psi|^{2} u d t d a d x .
\end{aligned}
$$

This computation gives twelve terms denoted by $I_{i, j}, i=1, \ldots, 4, j=1,2,3$.

We have by integration by parts

$$
I_{1,1}=\int_{Q} \frac{\partial u}{\partial t} \Delta u d t d a d x=\int_{\Sigma} \frac{\partial u}{\partial t} \frac{\partial u}{\partial \nu} d t d a d \sigma-\frac{1}{2} \int_{Q} \frac{\partial}{\partial t}|\nabla u|^{2} d t d a d x
$$

Hence using (3.4) and (3.6) it follows that

$$
\begin{gathered}
I_{11}=0 \\
I_{1,2}=s \int_{Q} \frac{\partial u}{\partial t}\left(\frac{\partial \eta}{\partial a}+\frac{\partial \eta}{\partial a}\right) u d t d a d x
\end{gathered}
$$

An integration by parts leads to

$$
\begin{aligned}
& I_{1,2}=-\frac{s}{2} \int_{Q}|u|^{2} \frac{\partial}{\partial t}\left(\frac{\partial \eta}{\partial a}+\frac{\partial \eta}{\partial a}\right) d t d a d x, \\
& I_{1,3}=s^{2} \lambda^{2} \int_{Q} \frac{\partial u}{\partial t} \varphi^{2} u|\nabla \Psi|^{2} d t d a d x .
\end{aligned}
$$

This gives

$$
I_{1,3}=\frac{s^{2} \lambda^{2}}{2} \int_{Q} \frac{\partial|u|^{2}}{\partial t} \varphi^{2}|\nabla \Psi|^{2} d t d a d x
$$

Keeping in mind (3.4), an integration by parts with respect to the variable $t$ yields

$$
I_{1,3}=-s^{2} \lambda^{2} \int_{Q}|u|^{2} \frac{\partial \varphi}{\partial t} \varphi|\nabla \Psi|^{2} d t d a d x
$$


Similarly, one gets easily that

$$
\begin{aligned}
& I_{21}=0 \\
& I_{2,2}=-\frac{s}{2} \int_{Q}|u|^{2} \frac{\partial}{\partial a}\left(\frac{\partial \eta}{\partial a}+\frac{\partial \eta}{\partial a}\right) d t d a d x, \\
& I_{2,3}=-s^{2} \lambda^{2} \int_{Q} \varphi|u|^{2} \frac{\partial \varphi}{\partial a}|\nabla \Psi|^{2} d t d a d x .
\end{aligned}
$$

Now, we are concerned by the term $I_{3, j}$.

We have

$$
I_{3,1}=-2 s \lambda \int_{Q} \varphi \nabla \Psi \cdot \nabla u \Delta u d t d a d x
$$

Then we have by an integration by parts

$$
I_{3,1}=-2 s \lambda \int_{\Sigma} \varphi \nabla \Psi \cdot \nabla u \frac{\partial u}{\partial \nu} d t d a d \sigma+2 s \lambda \int_{Q} \nabla u \cdot \nabla(\varphi \nabla \Psi \cdot \nabla u) d t d a d x .
$$

From the definition of $\Psi$ and since (3.6) is fulfilled we see that for all $\sigma \in \partial \Omega$ we have $\nabla u(t, a, \sigma)=(\nabla u(t, a, \sigma) \cdot \nu(\sigma)) \nu(\sigma)$ and $\nabla \Psi(\sigma)=(\nabla \Psi(\sigma) \cdot \nu(\sigma)) \nu(\sigma)$.

Therefore it follows, using also (3.8), that

$$
\begin{aligned}
I_{3,1}= & -2 s \lambda \int_{\Sigma} \varphi(\nabla \Psi \cdot \nu)|\nabla u \cdot \nu|^{2} d t d a d \sigma+2 s \lambda^{2} \int_{Q}|\nabla u \cdot \nabla \Psi|^{2} \varphi d t d a d x \\
& +2 s \lambda \sum_{i, j=1}^{N}\left(\int_{Q} \varphi \frac{\partial u}{\partial x_{i}} \frac{\partial^{2} u}{\partial x_{i} \partial x_{j}} \frac{\partial \Psi}{\partial x_{j}} d t d a d x+\int_{Q} \varphi \frac{\partial u}{\partial x_{i}} \frac{\partial^{2} \Psi}{\partial x_{i} \partial x_{j}} \frac{\partial u}{\partial x_{j}} d t d a d x\right) .
\end{aligned}
$$

We have

$$
\begin{aligned}
2 s \lambda \Sigma_{i, j=1}^{N} & \int_{Q} \varphi \frac{\partial u}{\partial x_{i}} \frac{\partial^{2} u}{\partial x_{i} \partial x_{j}} \frac{\partial \Psi}{\partial x_{j}} d t d a d x \\
= & s \lambda \int_{\Sigma} \varphi(\nabla \Psi \cdot n)|\nabla u \cdot n|^{2} d t d a d \sigma-s \lambda^{2} \int_{Q}|\nabla u|^{2}|\nabla \Psi|^{2} \varphi d t d a d x \\
& -s \lambda \int_{Q} \varphi|\nabla u|^{2} \Delta \Psi d t d a d x
\end{aligned}
$$

Therefore

$$
\begin{aligned}
I_{3,1}= & -s \lambda \int_{\Sigma} \varphi(\nabla \Psi \cdot n)|\nabla u \cdot \nu|^{2} d t d a d \sigma+2 s \lambda^{2} \int_{Q}|\nabla u \cdot \nabla \Psi|^{2} \varphi d t d a d x \\
& -s \lambda^{2} \int_{Q}|\nabla u|^{2}|\nabla \Psi|^{2} \varphi d t d a d x-s \lambda^{2} \int_{Q}|\nabla u|^{2}|\nabla \Psi|^{2} \varphi d t d a d x \\
& -s \lambda \int_{Q} \varphi|\nabla u|^{2} \Delta \Psi d t d a d x+2 s \lambda \Sigma_{i, j=1}^{N} \int_{Q} \varphi \frac{\partial^{2} \Psi}{\partial x_{i} \partial x_{j}} \frac{\partial u}{\partial x_{j}} \frac{\partial u}{\partial x_{i}} d t d a d x \\
I_{3,2}= & -2 s^{2} \lambda \int_{Q} \varphi \nabla \Psi \cdot \nabla u\left(\frac{\partial \eta}{\partial t}+\frac{\partial \eta}{\partial a}\right) u d t d a d x .
\end{aligned}
$$


8 Controllability of a nonlinear population model

Classical computations give

$$
\begin{aligned}
& I_{3,2}=-s^{2} \lambda^{2} \int_{Q} \varphi|\nabla \psi|^{2}|u|^{2}\left(\frac{\partial \eta}{\partial t}+\frac{\partial \eta}{\partial a}\right) d t d a d x+s^{2} \lambda \int_{Q} \varphi|u|^{2} \nabla \cdot\left(\nabla \Psi\left(\frac{\partial \eta}{\partial t}+\frac{\partial \eta}{\partial a}\right)\right), \\
& I_{3,3}=-2 s^{3} \lambda^{3} \int_{Q} \varphi^{3} \nabla \Psi \cdot \nabla u|\nabla \Psi|^{2} u d t d a d x .
\end{aligned}
$$

Equality (3.8) and an integration by part give

$$
I_{3,3}=3 s^{3} \lambda^{4} \int_{Q} \varphi^{3} u^{2}|\nabla \Psi|^{4} d t d a d x+s^{3} \lambda^{3} \int_{Q} \varphi^{3}|u|^{2} \nabla \cdot\left(\nabla \Psi|\nabla \Psi|^{2}\right) d t d a d x .
$$

Now we compute the terms $I_{4, j}$

$$
I_{4,1}=-2 s \lambda^{2} \int_{Q} \varphi u|\nabla \Psi|^{2} \Delta u d t d a d x=2 s \lambda^{2} \int_{Q} \nabla\left(\varphi u|\nabla \Psi|^{2}\right) \cdot \nabla u d t d a d x .
$$

Therefore

$$
\begin{aligned}
I_{4,1}= & 2 s \lambda^{3} \int_{Q} \varphi u \nabla \Psi \cdot \nabla u|\nabla \Psi|^{2} d t d a d x+2 s \lambda^{2} \int_{Q} \varphi|\nabla u|^{2}|\nabla \Psi|^{2} \nabla u d t d a d x \\
& +2 s \lambda^{2} \int_{Q} \varphi u \nabla u \cdot \nabla\left(|\nabla \Psi|^{2}\right) d t d a d x .
\end{aligned}
$$

Directly, we have

$$
\begin{aligned}
& I_{42}=-2 s^{2} \lambda^{2} \int_{Q} \varphi|\nabla \Psi|^{2}\left(\frac{\partial \eta}{\partial t}+\frac{\partial \eta}{\partial a}\right)|u|^{2} d t d a d x \\
& I_{43}=-2 s^{3} \lambda^{4} \int_{Q} \varphi^{3}|\nabla \Psi|^{4} u^{2} d t d a d x .
\end{aligned}
$$

Grouping all the terms $I_{i, j}$ and using the boundeness of the derivatives of $\varphi$ and $\eta$ one can write

$$
\begin{aligned}
2 \int_{Q} P_{1} u P_{2} u d t d a d x= & X_{1}+X_{2}-2 s \lambda \int_{\Sigma} \varphi \nabla \Psi \cdot v|\nabla u \cdot v|^{2} d t d a d \sigma \\
& +4 s \lambda^{2} \int_{Q} \varphi|\nabla u \cdot \nabla \Psi|^{2} d t d a d x \\
& +2 s \lambda^{2} \int_{Q} \varphi|\nabla u|^{2}|\nabla \Psi|^{2} d t d a d x+2 s^{3} \lambda^{4} \int_{Q} \varphi^{3} u^{2}|\nabla \Psi|^{4} d t d a d x
\end{aligned}
$$

where $X_{1}$ and $X_{2}$ verify

$$
\begin{aligned}
& X_{1} \leq C\left(s \lambda+\lambda^{2}\right) \int_{Q} \varphi|\nabla u|^{2} d t d a d x \\
& X_{2} \leq C\left(s^{2} \lambda^{4}+s^{3} \lambda^{3}\right) \int_{Q} \varphi^{3}|u|^{2} d t d a d x .
\end{aligned}
$$


Note that $\nu$ is the outward normal vector to $\partial \Omega$. So, using the fact that $\Psi(x)>0$ for all $x \in \Omega$ and $\Psi(\sigma)=0$ for all $\sigma \in \partial \Omega$ we infer that $\nabla \Psi \cdot \nu<0$. Therefore, (3.34) yields

$$
\begin{aligned}
2 \int_{Q} P_{1} u P_{2} u d t d a d x \geq & X_{1}+X_{2}+2 s \lambda^{2} \int_{Q} \varphi|\nabla u|^{2}|\nabla \Psi|^{2} d t d a d x \\
& +2 s^{3} \lambda^{4} \int_{Q} \varphi^{3} u^{2}|\nabla \Psi|^{4} d t d a d x .
\end{aligned}
$$

Note also that $\Psi \in C^{2}(\bar{\Omega})$ and $|\nabla \Psi| \neq 0$ in $\overline{\Omega-\widetilde{\omega}}$. Consequently, there exists a positive constant $\delta$ such that $|\nabla \Psi|>\delta$ in $\overline{\Omega-\widetilde{\omega}}$. Therefore (3.36) gives

$$
\begin{gathered}
2 \int_{Q} P_{1} u P_{2} u d t d a d x+2 s \lambda^{2} \delta^{2} \int_{\widetilde{q}} \varphi|\nabla u|^{2} d t d a d x+2 s^{3} \lambda^{4} \delta^{4} \int_{\widetilde{q}} \varphi^{3} u^{2} d t d a d x \\
\geq X_{1}+X_{2}+2 s \lambda^{2} \delta^{2} \int_{Q} \varphi|\nabla u|^{2} d t d a d x+2 s^{3} \lambda^{4} \delta^{4} \int_{Q} \varphi^{3} u^{2} d t d a d x
\end{gathered}
$$

where $\tilde{q}=(0, T) \times(0, A) \times \widetilde{\omega}$. Furthermore, we have

$$
\int_{Q} g_{s}^{2} d t d a d x \leq \int_{Q} e^{-2 s \eta} f^{2} d t d a d x+X_{1}+X_{2} .
$$

Then, it follows from (3.15) and (3.37) that

$$
\begin{aligned}
& \int_{Q} e^{-2 s \eta} f^{2} d t d a d x+X_{1}+X_{2}+2 s^{3} \lambda^{4} \delta^{4} \int_{\widetilde{q}} \varphi^{3}|u|^{2} d t d a d x+2 s \lambda^{2} \delta^{2} \int_{\widetilde{q}} \varphi|\nabla u|^{2} d t d a d x \\
& \geq \int_{Q}\left|P_{1} u\right|^{2} d t d a d x+\int_{Q}\left|P_{2} u\right|^{2} d t d a d x+2 s \lambda^{2} \delta^{2} \int_{Q} \varphi|\nabla u|^{2} d t d a d x \\
& \quad+2 s^{3} \lambda^{4} \delta^{4} \int_{Q} \varphi^{3}|u|^{2} d t d a d x .
\end{aligned}
$$

We can choose $s$ and $\lambda$ sufficiently large so that

$$
s \lambda^{2} \delta^{2} \int_{Q} \varphi|\nabla u|^{2} d t d a d x+s^{3} \lambda^{4} \delta^{4} \int_{Q} \varphi^{3}|u|^{2} d t d a d x \geq X_{1}+X_{2} .
$$

This means more precisely that there exists positive constants $s_{1}>1$ and $\lambda_{1}>1$ such that for $s \geq s_{1}$ and $\lambda \geq \lambda_{1}$ (3.39) yields

$$
\begin{aligned}
& \int_{Q} e^{-2 s \eta} f^{2} d t d a d x+2 s^{3} \lambda^{4} \delta^{4} \int_{\widetilde{q}} \varphi^{3}|u|^{2} d t d a d x+2 s \lambda^{2} \delta^{2} \int_{\tilde{q}} \varphi|\nabla u|^{2} d t d a d x \\
& \geq \int_{Q}\left|P_{1} u\right|^{2} d t d a d x+\int_{Q}\left|P_{2} u\right|^{2} d t d a d x+s \lambda^{2} \delta^{2} \int_{Q} \varphi|\nabla u|^{2} d t d a d x \\
& \quad+s^{3} \lambda^{4} \delta^{4} \int_{Q} \varphi^{3}|u|^{2} d t d a d x .
\end{aligned}
$$


10 Controllability of a nonlinear population model

We want now to eliminate the term

$$
2 s \lambda^{2} \delta^{2} \int_{\widetilde{q}} \varphi|\nabla u|^{2} d t d a d x
$$

in (3.41). For this aim, we introduce a cut-off function $\alpha$ such that $\alpha \in C_{0}^{\infty}(\omega) ; 0 \leq \alpha \leq 1$; and $\alpha=1$ on $\tilde{\omega}$.

Multiplying $P_{2} u$ by $\varphi \alpha^{2} u$ and integrating the result over $Q$ leads to

$$
\begin{aligned}
& \int_{Q} \varphi \alpha^{2} u P_{2} u d t d a d x \\
& \quad=-s \int_{Q}\left(\frac{\partial \eta}{\partial t}+\frac{\partial \eta}{\partial a}\right) u^{2} \varphi \alpha^{2} d t d a d x-s^{2} \lambda^{2} \int_{Q} u^{2} \varphi^{3} \alpha^{2}|\Psi|^{2} d t d a d-\int_{Q} u \Delta u \varphi \alpha^{2} d t d a d x
\end{aligned}
$$

Note that

$$
\begin{aligned}
\int_{Q} u \Delta & u \varphi \alpha^{2} d t d a d x \\
& =-\int_{Q}|\nabla u|^{2} \varphi \alpha^{2} d t d a d x-\lambda \int_{Q} u \nabla u \cdot \nabla \Psi \varphi \alpha^{2} d t d a d x-2 \int_{Q} u \nabla u \cdot \nabla \alpha \varphi \alpha d t d a d x
\end{aligned}
$$

Therefore

$$
\begin{aligned}
\int_{Q} \varphi \alpha^{2} u P_{2} u d t d a d x= & -s \int_{Q}\left(\frac{\partial \eta}{\partial t}+\frac{\partial \eta}{\partial a}\right) u^{2} \varphi \alpha^{2} d t d a d x \\
& -s^{2} \lambda^{2} \int_{Q} u^{2} \varphi^{3} \alpha^{2}|\Psi|^{2} d t d a d x+\int_{Q}|\nabla u|^{2} \varphi \alpha^{2} d t d a d x \\
& +\lambda \int_{Q} u \nabla u \cdot \nabla \Psi \varphi \alpha^{2} d t d a d x+2 \int_{Q} u \nabla u \cdot \nabla \alpha \varphi \alpha d t d a d x
\end{aligned}
$$

This gives

$$
\begin{aligned}
\int_{Q}|\nabla u|^{2} \varphi \alpha^{2} d t d a d x= & \int_{Q} \varphi \alpha^{2} u P_{2} u d t d a d x+s \int_{Q}\left(\frac{\partial \eta}{\partial t}+\frac{\partial \eta}{\partial a}\right) u^{2} \varphi \alpha^{2} d t d a d x \\
& +s^{2} \lambda^{2} \int_{Q} u^{2} \varphi^{3} \alpha^{2}|\Psi|^{2} d t d a d x-\lambda \int_{Q} u \nabla u \cdot \nabla \Psi \varphi \alpha^{2} d t d a d x \\
& -2 \int_{Q} u \nabla u \cdot \nabla \alpha \varphi \alpha d t d a d x .
\end{aligned}
$$

Note that

$$
-\lambda \int_{Q} u \nabla u \cdot \nabla \Psi \varphi \alpha^{2} d t d a d x \leq C \lambda^{2} \int_{Q}|u|^{2} \varphi \alpha^{2} d t d a d x+\frac{1}{2} \int_{Q}|\nabla u|^{2} \varphi \alpha^{2} d t d a d x
$$


where $C$ is a positive constant. As $\varphi \leq C \varphi^{3}$ with $C$ a positive constant, using now the properties of $\alpha$ and $\Psi$ we deduce

$$
\begin{aligned}
& \int_{\widetilde{q}}|\nabla u|^{2} \varphi \alpha^{2} d t d a d x \\
& \quad \leq C \int_{Q} \varphi \alpha^{2} u P_{2} u d t d a d x+C s^{2} \lambda^{2} \int_{Q} u^{2} \varphi^{3} \alpha^{2} d t d a d x+C \int_{Q} u \varphi^{1 / 2}|\nabla u| \varphi^{1 / 2} \alpha d t d a d x .
\end{aligned}
$$

Therefore we deduce from the previous estimate that

$$
2 s \lambda^{2} \delta^{2} \int_{\widetilde{q}}|\nabla u|^{2} \varphi d t d a d x \leq \frac{1}{2} \int_{Q}\left|P_{2} u^{2}\right| d t d a d x+C s^{2} \lambda^{2} \int_{q} u^{2} \varphi^{3} d t d a d x,
$$

where $C$ is a positive constant.

Combining (3.41) and (3.49) we get

$$
\begin{aligned}
& C\left(\int_{Q} e^{-2 s \eta} f^{2} d t d a d x+s^{3} \lambda^{4} \int_{q} \varphi^{3} u^{2} d t d a d x\right) \\
& \quad \geq \int_{Q}\left|P_{1} u\right|^{2} d t d a d x+\int_{Q}\left|P_{2} u\right|^{2} d t d a d x+s \lambda^{2} \int_{Q} \varphi|\nabla u|^{2} d t d a d x \\
& \quad+s^{3} \lambda^{4} \int_{Q} \varphi^{3} u^{2} d t d a d x
\end{aligned}
$$

We want now to turn back to the variable $w$.

Note that $u=e^{-s \eta} w$. Then, we have

$$
\begin{aligned}
& \int_{Q} \varphi^{3}|u|^{2} d t d a d x=\int_{Q} e^{-2 s \eta} \varphi^{3}|w|^{2} d t d a d x \\
& \int_{q} \varphi^{3}|u|^{2} d t d a d x=\int_{q} e^{-2 s \eta} \varphi^{3}|w|^{2} d t d a d x
\end{aligned}
$$

Therefore one gets from (3.50)

$$
s^{3} \lambda^{4} \int_{Q} \varphi^{3} e^{-2 s \eta} w^{2} d t d a d x \leq C \int_{Q} e^{-2 s \eta} f^{2} d t d a d x+C s^{3} \lambda^{4} \int_{q} e^{-2 s \eta} \varphi^{3} w^{2} d t d a d x .
$$

This ends the proof.

Remark 3.3. (i) Indeed one can prove that there exist positive constants $s_{1} \geq 1$ and $\lambda_{1} \geq 1$ and there exists a positive constant $C>0$ such that for all $s \geq s_{1}, \lambda \geq \lambda_{1}$ and for all solution 
of (3.1) the following inequality holds:

$$
\begin{aligned}
& \int_{Q} \frac{e^{-2 s \eta}}{s \varphi}\left(\left|\frac{\partial w}{\partial t}+\frac{\partial w}{\partial a}\right|^{2}+|\Delta w|^{2}\right) d x d a d t+\int_{Q} e^{-2 s \eta} s^{3} \varphi^{3} \lambda^{4} w^{2} d t d a d x \\
& \quad+\int_{Q} e^{-2 s \eta} s \lambda \varphi|\nabla w|^{2} d t d a d x \leq C\left(\int_{Q} e^{-2 s \eta} f^{2} d t d a d x+\int_{q} e^{-2 s \eta} s^{3} \varphi^{3} \lambda^{4} w^{2} d t d a d x\right) .
\end{aligned}
$$

It is sufficient to use (3.50) and to turn back to the variable $w$ by using the explicit expression of $P_{1} u$ and $P_{2} u$.

(ii) In [1] the author tried to prove a Carleman inequality for the system (3.1) with $\beta w(t, 0, x)$ instead of $f$. The problem there is more complex: after the change of variable $u=e^{-2 s \eta} w$ the right term becomes $e^{-2 s \eta} w(t, 0, x)$ and cannot be written in terms of the variable $u$. Unfortunately, see [1, system (6) page 566], this term was ignored in the computations.

In the sequel we take $f=0$ in order to avoid this situation.

Our observability inequality is as follows.

Proposition 3.4. Assume that

$$
f=0
$$

and that there exists a real $\gamma \geq 0$ such that

$$
g(a, x)=0 \quad \text { a.e. in }(0, \gamma) \times \Omega .
$$

Then, there exists a positive constant $C_{\gamma}$ such that the following inequality holds:

$$
\int_{Q_{A}} w^{2}(0, a, x) d a d x+\int_{Q_{T}} w^{2}(t, 0, x) d t d x \leq C_{\gamma} \int_{q} w^{2}(t, a, x) d t d a d x
$$

for all solution $w$ of (3.1).

Let $\gamma$ be small enough so that $\gamma \leq \min (T, A)$. We define now two subsets of $(0, T) \times$ $(0, A)$ :

$$
\begin{aligned}
& N_{1}=\{(t, a) \in(0, T) \times(0, A) ; t \geq a+T-\gamma\}, \\
& N_{2}=\{(t, a) \in(0, T) \times(0, A) ; t \leq a+\gamma-A\},
\end{aligned}
$$

and we formulate a lemma which will be used in the proof of Proposition 3.4.

LEMMA 3.5. If (3.54) and (3.55) hold, then all solutions of (3.1) verify

$$
w(t, a, x)=0 \quad \text { a.e. in }\left(N_{1} \cup N_{2}\right) \times \Omega \text {. }
$$


Proof of Lemma 3.5. We will prove that $w=0$ on almost every characteristic line in $N_{1} \cup$ $\mathrm{N}_{2}$.

Let $\left(t_{0}, a_{0}\right) \in N_{1}$. Then we have $t_{0}=a_{0}+T-\gamma+d$ with $0 \leq d \leq \gamma$. Therefore, $a_{0} \leq$ $\gamma-d$.

Let $S(d)=\left\{\left(t_{0}+s, a_{0}+s\right), s \in\left(0, \gamma-d-a_{0}\right)\right\}$ be a characteristic line of (3.1). Setting $z(s, x)=w\left(t_{0}+s, a_{0}+s, x\right)$ and $\bar{\mu}(s, x)=\mu\left(t_{0}+s, a_{0}+s, x\right)$ from (3.1), we deduce that $z$ solves

$$
\begin{gathered}
-\frac{\partial z}{\partial s}-\triangle z+\bar{\mu} z=0 \quad \text { in }\left(0, \gamma-d-a_{0}\right) \times \Omega, \\
z(s, x)=0 \quad \text { on }\left(0, \gamma-d-a_{0}\right) \times \partial \Omega, \\
z\left(\gamma-d-a_{0}, x\right)=w(T, \gamma-d, x)=g(\gamma-d, x) \quad \text { in } \Omega .
\end{gathered}
$$

Then from (3.55) for almost all $d \in(0, \gamma)$, standard results on heat equation imply that $z=0$. Thus, for almost all $d \in(0, \gamma), w=0$ on $S(d)$. Therefore, $w=0$ in $N_{1} \times \Omega$. The same argument and the fact that $w(t, A, x)=0$ in $(0, T) \times \Omega$ allow us to prove that $w=0$ in $N_{2} \times \Omega$.

Now, let us prove Proposition 3.4.

Proof of Proposition 3.4. We set

$$
\begin{aligned}
& D_{1}=\left\{(t, a) \in(0, T) \times(0, A), t \leq-\frac{T-\gamma / 2}{A-\gamma / 2} a+T-\frac{\gamma}{2}\right\}, \\
& D_{2}=\left\{(t, a) \in(0, T) \times(0, A), a \geq-\frac{A-\gamma / 2}{T-\gamma / 2} t+A-\frac{\gamma(\gamma-2 A)}{2(2 T-\gamma)}\right\}, \\
& D_{3}=(0, T) \times(0, A)-\left(D_{1} \cup D_{2}\right), \\
& D_{4}=\left\{(t, a) \in D_{3} ;(t, a) \notin\left(N_{1} \cup N_{2}\right)\right\}, \quad \text { (cf. Figure 3.1). }
\end{aligned}
$$

Consider now $\theta \in C_{0}^{\infty}\left(\mathbb{R}^{2}\right)$ a cut-off function such that $\theta=1$ on $D_{1} ; \theta=0$ on $D_{2}$. Setting $\widetilde{w}=\theta w$, it follows that $\widetilde{w}$ solves

$$
\begin{aligned}
-\frac{\partial \widetilde{w}}{\partial t}-\frac{\partial \widetilde{w}}{\partial a}-\triangle \widetilde{w}+\mu \tilde{w} & =-\left(\frac{\partial \theta}{\partial t}+\frac{\partial \theta}{\partial a}\right) w \quad \text { in } Q \\
\widetilde{w}(t, a, x) & =0 \quad \text { on } \Sigma \\
\widetilde{w}(T, a, x) & =0 \quad \text { in } Q_{A}, \\
\widetilde{w}(t, A, x) & =0 \quad \text { in } Q_{T} .
\end{aligned}
$$

Multiplying (3.61) by $\widetilde{w}$ and integrating over $Q$ yield after minor majoration

$$
\int_{0}^{T-\gamma / 2} \int_{\Omega} w^{2}(t, 0, x) d x d t+\int_{0}^{A-\gamma / 2} \int_{\Omega} w^{2}(0, a, x) d x d a \leq-2 \int_{Q}\left(\frac{\partial \theta}{\partial t}+\frac{\partial \theta}{\partial a}\right) \theta w^{2} d t d a d x .
$$


14 Controllability of a nonlinear population model

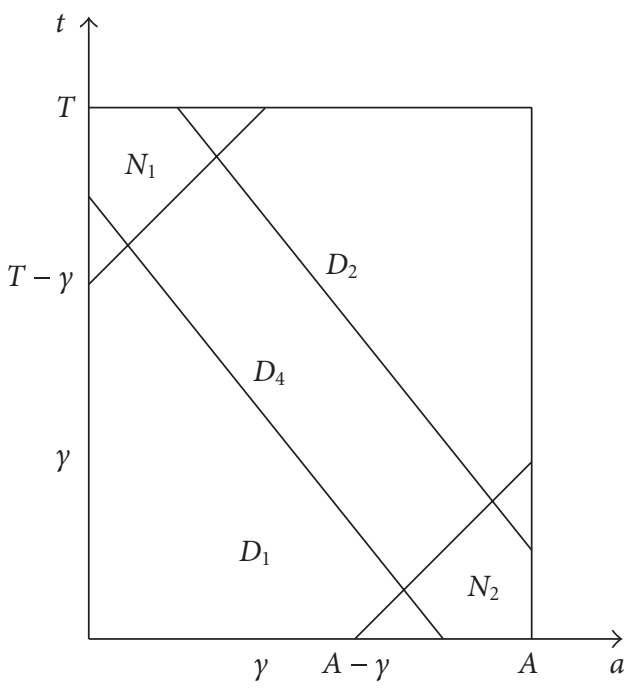

Figure 3.1

Using Lemma 3.5 and the definition of $\theta$, we deduce that $(\partial \theta / \partial t+\partial \theta / \partial a) \theta w=0$ almost every where outside of $D_{4} \times \Omega$. Note that $\eta$ and $\varphi$ are bounded on $D_{4} \times \Omega$ by strictly positive reals. Hence there exists a positive constant $\bar{C}_{\gamma}>0$ such that

$$
-2 \int_{Q}\left(\frac{\partial \theta}{\partial t}+\frac{\partial \theta}{\partial a}\right) \theta w^{2} d t d a d x \leq \bar{C}_{\gamma} \int_{Q} \varphi^{2} e^{-2 s \eta} w^{2} d t d a d x
$$

Therefore (3.62) yields

$$
\int_{0}^{T-(\gamma / 2)} \int_{\Omega} w^{2}(t, 0, x) d x d t+\int_{0}^{A-(\gamma / 2)} \int_{\Omega} w^{2}(0, a, x) d x d a \leq \bar{C}_{\gamma} \int_{Q} \varphi^{2} e^{-2 s \eta} w^{2} d t d a d x
$$

where $\bar{C}_{\gamma}$ is a positive constant depending on $\gamma$. Using now (3.2), (3.58) and the fact that $\varphi^{2} e^{-2 s \eta} \leq 1$ for $\lambda$ and $s$ sufficiently large we deduce (3.56).

Remark 3.6. A careful calculation for $s \geq s_{1}$ and $\lambda \geq \lambda_{1}$ leads to the following estimate of $C_{\gamma}$ :

$$
C_{\gamma} \geq C(T) \gamma^{2} \exp \left(\frac{C(\Psi, s, \lambda)}{\gamma^{3} A T}\right)
$$

where $C(\Psi, s, \lambda)$ and $C(T)$ are positive constants. 
3.2. A null controllability result. In this section, for a given function $b \in L^{2}\left(Q_{T}\right)$ we consider the following system:

$$
\begin{gathered}
\frac{\partial y}{\partial t}+\frac{\partial y}{\partial a}-\Delta y+\mu y=v 1_{\omega} \quad \text { in } Q, \\
y(t, a, \sigma)=0 \quad \text { on } \Sigma, \\
y(0, a, x)=y_{0}(a, x) \quad \text { in } Q_{A}, \\
y(t, 0, x)=b(t, x) \quad \text { in } Q_{T} .
\end{gathered}
$$

For all $\epsilon>0$ we introduce the functional

$$
J_{\epsilon}(v)=\frac{1}{2 \epsilon} \int_{\gamma}^{A} \int_{\Omega} y^{2}(T, a, x) d x d a+\frac{1}{2} \int_{q} v^{2}(t, a, x) d x d a d t .
$$

It follows easily that $J_{\epsilon}$ is continuous, convex, and coercive. Hence, $J_{\epsilon}$ admits a unique minimizer $v_{\epsilon}$ and we have

$$
v_{\epsilon}(t, a, x)=-w_{\epsilon}(t, a, x) 1_{\omega}(x) \text { in } Q
$$

where $w_{\epsilon}$ is the solution of the following system:

$$
\begin{gathered}
-\frac{\partial w_{\epsilon}}{\partial t}-\frac{\partial w_{\epsilon}}{\partial a}-\Delta w_{\epsilon}+\mu w_{\epsilon}=0 \quad \text { in } Q, \\
w_{\epsilon}(t, a, \sigma)=0 \quad \text { on } \Sigma, \\
w_{\epsilon}(T, a, x)=\frac{1}{\epsilon} y_{\epsilon}(T, a, x) 1_{(\gamma, A)}(a) \text { in } Q_{A}, \\
w_{\epsilon}(t, A, x)=0 \quad \text { in } Q_{T},
\end{gathered}
$$

and $y_{\epsilon}$ is the solution of (3.66) associated to $v_{\epsilon}$.

Multiplying (3.69) by $y_{\epsilon}$ and integrating on $Q$ give

$$
\begin{gathered}
-\frac{1}{\epsilon} \int_{\gamma}^{A} \int_{\Omega} y_{\epsilon}^{2}(T, a, x) d x d a+\int_{0}^{A} \int_{\Omega} w_{\epsilon}(0, a, x) y_{0}(a, x) d x d a \\
\quad+\int_{0}^{T} \int_{\Omega} w_{\epsilon}(t, 0, x) b(t, x) d x d t+\int_{q} v_{\epsilon} w_{\epsilon} d t d a d x=0 .
\end{gathered}
$$

Using (3.68) we obtain

$$
\begin{gathered}
\int_{0}^{A} \int_{\Omega} w_{\epsilon}(0, a, x) y_{0}(a, x) d x d a+\int_{0}^{T} \int_{\Omega} w_{\epsilon}(t, 0, x) b(t, x) d x d t \\
=\frac{1}{\epsilon} \int_{\gamma}^{A} \int_{\Omega} y_{\epsilon}^{2}(T, a, x) d x d a+\int_{q} v_{\epsilon}^{2} d t d a d x .
\end{gathered}
$$


On the other hand, Young inequality gives

$$
\begin{aligned}
\int_{0}^{A} \int_{\Omega} w_{\epsilon}(0, a, x) y_{0}(a, x) d x d a+\int_{0}^{T} \int_{\Omega} w_{\epsilon}(t, 0, x) b(t, x) d x d t \\
\leq \frac{1}{2 C_{\gamma}}\left(\int_{0}^{A} \int_{\Omega} w_{\epsilon}^{2}(0, a, x) d x d a+\int_{0}^{T} \int_{\Omega} w_{\epsilon}^{2}(t, 0, x) d t d x\right) \\
\quad+2 C_{\gamma}\left(\int_{0}^{A} \int_{\Omega} y_{0}^{2}(a, x) d x d a+\int_{0}^{T} \int_{\Omega} b^{2}(t, x) d x d t\right) .
\end{aligned}
$$

Therefore Proposition 3.4 and inequality (3.72) imply

$$
\begin{aligned}
& \frac{1}{\epsilon} \int_{\gamma}^{A} \int_{\Omega} y_{\epsilon}^{2}(T, a, x) d x d a+\frac{1}{2} \int_{q} v_{\epsilon}^{2} d t d a d x \\
& \quad \leq 2 C_{\gamma}\left(\int_{0}^{A} \int_{\Omega} y_{0}^{2}(a, x) d x d a+\int_{0}^{T} \int_{\Omega} b^{2}(t, x) d x d t\right) .
\end{aligned}
$$

Consequently

$$
\begin{gathered}
\left\|v_{\epsilon}\right\|_{L^{2}(q)}^{2} \leq 4 C_{\gamma}\left(\|b\|_{L^{2}\left(Q_{T}\right)}^{2}+\left\|y_{0}\right\|_{L^{2}\left(Q_{A}\right)}^{2}\right), \\
\int_{\Omega} y_{\epsilon}^{2}(T, a, x) d x d a \leq 2 \epsilon C_{\gamma}\left(\|b\|_{L^{2}\left(Q_{T}\right)}^{2}+\left\|y_{0}\right\|_{L^{2}\left(Q_{A}\right)}^{2}\right) .
\end{gathered}
$$

Then, one can extract subsequences also denoted by $v_{\epsilon}$ and $y_{\epsilon}$ such that $v_{\epsilon} \rightarrow v$ weakly in $L^{2}(q)$ and $y_{\epsilon} \rightarrow y$ weakly in $L^{2}\left((0, T) \times(0, A), H_{0}^{1}(\Omega)\right)$.

Moreover $y$ is the unique solution of (3.66) and verifies (2.2). Notice also that $v$ verifies (2.2).

Therefore, we have proved the following null controllability result.

Proposition 3.7. For any given positive real $\gamma$ small enough, there exists a control $v \in L^{2}(q)$ that verifies (3.74), such that the associated solution y of (3.66) verifies (2.2).

Remark 3.8. (i) This result is quite similar to what was proved in [7] for a so-called "linearized crocco-type equation." More precisely, it was proved in [7] that there exists a control $v$ acting on $\left(x_{0}, x_{1}\right) \times \omega$, with $0<x_{0}<x_{1}<A$ such that the corresponding solution of (3.66) with $\Omega \subset \mathbb{R}$ verifies

$$
y(T, a, x)=0 \quad \text { in }\left(x_{0}+\delta, L\right) \times \Omega,
$$

where

$$
L= \begin{cases}x_{1}+T-\delta & \text { if } 0<T<A-x_{1}+\delta \\ A & \text { if } T>A-x_{1}+\delta .\end{cases}
$$

See [7, page 710].

The method in [7] uses the fact that $0<x_{0}<A$, energy estimates, and Carleman estimates for parabolic equation along characteristic lines of (3.66). Therefore one cannot use the result of [7] for the case $x_{0}=0$ and $x_{1}=A$ which is studied here. 
(ii) System (3.13) describes in fact the evolution of a controlled age and space structured population in which the birth process is given by a function regardless of the distribution of individuals of age $a>0$. That explains why it seems impossible to eradicate individuals of age close to 0 .

\section{Proof of the main result}

For $\theta \in L^{2}\left(Q_{T}\right)$, letting $b=e^{-\lambda_{0} t} F\left(e^{\lambda_{0} t} \theta\right)$, we derive from Proposition 3.7 that there exists a control $v$ that verifies (3.74) so that the corresponding solution of (3.66) verifies (2.2). Then for all $\theta \in L^{2}\left(Q_{T}\right)$ we define by $\Lambda(\theta)$ the nonempty set of all $\int_{0}^{A} \beta y d a$ where $y$ verifies (2.2), solves (3.66) with $v \in L^{2}(q)$ that verifies (3.74). The problem is now reduced to find a fixed point for $\Lambda$. In order to apply a generalization of the Leray-Schauder fixed point theorem stated in [5], we define the set $N=\left\{\theta \in L^{2}\left(Q_{T}\right),(\exists) \zeta \in(0,1), \theta \in \zeta \Lambda(\theta)\right\}$. Thus doing the existence of a fixed point is a obvious consequence of the following.

Proposition 4.1. (i) $\Lambda$ is a compact multivalued mapping of $L^{2}\left(Q_{T}\right)$.

(ii) For all $\theta \in L^{2}\left(Q_{T}\right), \Lambda(\theta)$ is a nonempty closed convex subset of $L^{2}\left(Q_{T}\right)$.

(iii) $N$ is bounded in $L^{2}\left(Q_{T}\right)$.

(iv) $\Lambda$ is upper semicontinuous on $L^{2}\left(Q_{T}\right)$.

Proof of Proposition 4.1. (i) We prove the compactness of $\Lambda$. Let $\theta \in L^{2}\left(Q_{T}\right)$ such that $\|\theta\| \leq r, r>0$. We have to prove that $\Lambda(\theta)$ is compact in $L^{2}\left(Q_{T}\right)$. Consider $\left(\rho_{n}\right)_{n} \subset$ $\Lambda(\theta)$. From the definition of $\Lambda$, for all $n$ there exists a pair $\left(v_{n}, y_{n}\right) \in L^{2}(q) \times L^{2}(Q)$ such that $\rho_{n}=\int_{0}^{A} \beta y_{n} d a, v_{n}$ verifies (3.74) and $y_{n}$, the associated solution of (3.66) with $b=e^{-\lambda_{0} t} F\left(e^{\lambda_{0} t} \theta\right)$ verifies $(2.2)$.

Using (3.74) we deduce that

$$
\left\|v_{n}\right\|_{L^{2}(q)}^{2} \leq 4 C_{\gamma}\left(\left\|e^{-\lambda_{0} t} F\left(e^{\lambda_{0} t} \theta\right)\right\|_{L^{2}\left(Q_{T}\right)}^{2}+\left\|y_{0}\right\|_{L^{2}\left(Q_{A}\right)}^{2}\right) .
$$

Then we get via $\mathrm{H}_{3}$

$$
\left\|v_{n}\right\|_{L^{2}(q)}^{2} \leq C_{\gamma}\left(C(F, \Omega, T, r)+\left\|y_{0}\right\|_{L^{2}\left(Q_{A}\right)}^{2}\right)
$$

Multiplying (3.66) with $e^{-\lambda_{0} t} F\left(e^{\lambda_{0} t} \theta\right)$ instead of $b$ by $y_{n}$ and integrating over $Q$, we obtain

$$
\left\|\nabla y_{n}\right\|_{L^{2}(Q)}^{2}+\frac{\lambda_{0}}{2}\left\|y_{n}\right\|_{L^{2}(Q)}^{2} \leq \frac{2}{\lambda_{0}}\left\|v_{n}\right\|_{L^{2}(q)}^{2}+\frac{1}{2}\left\|y_{0}\right\|_{L^{2}\left(Q_{A}\right)}^{2}+\frac{1}{2}\left\|e^{-\lambda_{0} t} F\left(e^{\lambda_{0} t} \theta\right)\right\|_{L^{2}\left(Q_{T}\right)}^{2} .
$$

Therefore, for $\lambda_{0} \geq 2$ we get

$$
\|\nabla y\|_{L^{2}(Q)}^{2}+\|y\|_{L^{2}(Q)}^{2} \leq\left(C_{\gamma}+1\right)\left(C(F, \Omega, r, T)+\left\|y_{0}\right\|_{L^{2}\left(Q_{A}\right)}^{2}\right) .
$$


Moreover, using $H_{2}$ we deduce that $\rho_{n}=\int_{0}^{A} \beta y_{n} d a$ solves the system

$$
\begin{gathered}
\frac{\partial \rho_{n}}{\partial t}-\Delta \rho_{n}+\int_{0}^{A} \beta \mu y_{n} d a=z_{n}(t, x) \text { in } Q_{T}, \\
\rho(t, x)=0 \quad \text { on }(0, T) \times \partial \Omega, \\
\rho_{n}(0, x)=\int_{0}^{A} \beta(0, a, x) y_{0}(a, x) d a \quad \text { in } \Omega,
\end{gathered}
$$

where $z_{n}(t, x)=\int_{0}^{A} \beta v_{n} d a 1_{\omega}+\int_{0}^{A} y_{n}(\partial \beta / \partial t+\partial \beta / \partial a-\triangle \beta) d a+\int_{0}^{A} \nabla y_{n} \nabla \beta d a$.

Notice that

$$
\left\|z_{n}\right\|_{L^{2}\left(Q_{T}\right)}^{2} \leq 3 C_{\beta}^{2} A\left(\left\|v_{n}\right\|_{L^{2}(q)}^{2}+\left\|y_{n}\right\|_{L^{2}(Q)}^{2}+\left\|\nabla y_{n}\right\|_{L^{2}(Q)}^{2}\right) .
$$

This implies via (4.2) and (4.4) that

$$
\left\|z_{n}\right\|_{L^{2}\left(Q_{T}\right)}^{2} \leq\left(C_{\gamma}+1\right) C(\beta, A)\left(C(F, \Omega, r, T)+\left\|y_{0}\right\|_{L^{2}\left(Q_{A}\right)}^{2}\right) .
$$

Now let us multiply (4.5) by $\rho_{n}$, we obtain after an integration by parts and minor changes that

$$
\left\|\nabla \rho_{n}\right\|_{L^{2}\left(Q_{T}\right)}^{2}+\frac{\lambda_{0}}{2}\left\|\rho_{n}\right\|_{L^{2}\left(Q_{T}\right)}^{2} \leq \frac{2}{\lambda_{0}}\left\|z_{n}\right\|_{L^{2}\left(Q_{A}\right)}^{2}
$$

Consequently, $\rho_{n}$ is bounded in $L^{2}\left((0, T), H_{0}^{1}(\Omega)\right)$ and standard arguments allow us to see that $\partial \rho_{n} \partial t$ is also bounded in $L^{2}\left((0, T), H_{0}^{-1}(\Omega)\right)$. Hence, using Lions-Aubin lemma we conclude the proof of (i).

We address now the proof of (ii).

First, it is obvious that for all $\theta \in L^{2}\left(Q_{T}\right), \Lambda(\theta)$ is a nonempty convex set. Let $\left(\rho_{n}\right)_{n} \subset$ $\Lambda(\theta)$ such that $\rho_{n} \rightarrow \rho$ in $L^{2}\left(Q_{T}\right)$. We have to prove that $\rho \in \Lambda(\theta)$. For all $n$ there exists $v_{n}$ that verifies (3.74) such that $\rho_{n}=\int_{0}^{A} \beta y_{n} d a$ where $y_{n}$ is the corresponding solution of (3.66) with $e^{\lambda_{0} t} F\left(e^{\lambda t} \theta\right)$ instead of $b$, and $y_{n}$ verifies also (2.2). Then, from (4.2) and (4.4) we deduce that one can extract subsequences also denoted by $v_{n}$ and $y_{n}$ converging weakly to $v$ and $y$, respectively, in $L^{2}(q)$ and $L^{2}\left((0, T) \times(0, A), H_{0}^{1}(\Omega)\right)$. Standard device implies that $\int_{0}^{A} \beta y d a=\rho$. In addition, it follows that $y$ is the associated solution of (3.66) with $b=e^{-\lambda_{0} t} F\left(e^{\lambda_{0} t} \theta\right)$. In addition $v$ verifies (3.74) and $y$ verifies (2.2). Therefore, the definition of $\Lambda$ yields that $\rho \in \Lambda(\theta)$.

Let us perform now the proof of (iii). Let $\theta \in N$, then there exists $\zeta \in(0,1)$ such that $(1 / \zeta) \theta \in \Lambda \theta$. As a consequence, there exists a pair $(\nu, y) \in L^{2}(q) \times L^{2}(Q)$ such that $\theta=$ $\zeta \int_{0}^{A} \beta y d a, v$ verifies (3.74) and $y$ is the associated solution of (3.66) with $b=e^{-\lambda_{0} t} F\left(e^{\lambda_{0} t} \theta\right)$. This implies on one hand that

$$
\|\theta\|_{L^{2}\left(Q_{T}\right)}^{2} \leq C(\beta, A)\|y\|_{L^{2}(Q)}^{2} .
$$

By (4.1) and $H_{3}$ we deduce

$$
\|v\|_{L^{2}(q)}^{2} \leq 8 C_{\gamma}\left(C\left(C_{0}, \Omega, T\right)+C_{1}^{2}\|\theta\|_{L^{2}\left(Q_{T}\right)}^{2}+\left\|y_{0}\right\|_{L^{2}\left(Q_{A}\right)}^{2}\right)
$$


and consequently, (4.3) yields

$$
\|y\|_{L^{2}(Q)}^{2} \leq \frac{16}{\lambda_{0}}\left(C\left(T, \Omega, C_{0}\right)+\left\|y_{0}\right\|_{L^{2}\left(Q_{A}\right)}^{2}\right)+\frac{\left(16 C_{\gamma}+1\right) C_{1}^{2}}{\lambda_{0}} \|\left.\theta\right|_{L^{2}\left(Q_{T}\right)} ^{2} .
$$

Taking now $\lambda_{0}>\max \left(2,\left(16 C_{\gamma}+1\right) C_{1}^{2}\right)$ and combining (4.9) and (4.11) we get

$$
\|\theta\|_{L^{2}\left(Q_{T}\right)}^{2} \leq C\left(A, T, \Omega, F, \gamma,\left\|y_{0}\right\|_{L^{2}\left(Q_{A}\right)}^{2}\right)
$$

that achieves the proof of (iii).

It remains to check that $\Lambda$ is upper semicontinuous on $L^{2}\left(Q_{T}\right)$. This is equivalent to prove that for any closed subset $G$ of $L^{2}\left(Q_{T}\right), \Lambda^{-1}(G)$ is closed in $L^{2}\left(Q_{T}\right)$. Let $\theta_{n} \in \Lambda^{-1}(G)$ such that $\theta_{n}$ converges towards $\theta$ in $L^{2}\left(Q_{T}\right)$. Then, $\theta_{n}$ is bounded and for all $n$ there exists $\rho_{n} \in G$ such that $\rho_{n} \in \Lambda\left(\theta_{n}\right)$. Therefore, from the definition of $\Lambda$ there exists a pair $\left(v_{n}, y_{n}\right) \in L^{2}(q) \times L^{2}(Q)$ such that $\rho_{n}=\int_{0}^{A} \beta y_{n} d a, v_{n}$ verifies (3.74), $y_{n}$ the corresponding solution of (3.66) with $e^{-\lambda_{0} t} F\left(e^{\lambda_{0} t} \theta_{n}\right)$ instead of $b$ verifies (2.2), so that $v_{n}$ verifies (4.2) and $y_{n}(4.4)$. Consequently $\left(v_{n}, y_{n}\right)$ is bounded in $L^{2}(q) \times L^{2}(Q)$. Thus, there exists a subsequence still denoted by $\left(v_{n}, y_{n}\right)$ that converges weakly to $(v, y)$ in $L^{2}(q) \times L^{2}(Q)$. Since $F$ is continuous, it follows that $e^{-\lambda_{0} t} F\left(e^{\lambda_{0} t} \theta_{n}\right)$ converges strongly towards $e^{-\lambda_{0} t} F\left(e^{\lambda_{0} t} \theta\right)$. Now, by standard device we see that $v$ verifies (3.74), $\rho=\int_{0}^{A} \beta y d a, y$ solves (3.66) with $e^{-\lambda_{0} t} F\left(e^{\lambda_{0} t} \theta\right)$ instead of $b$ and $y$ verifies in addition (2.2). This implies obviously that

$$
\rho \in \Lambda(\theta)
$$

On the other hand, thanks to (4.8) and Lions-Aubin lemma once again, one can extract a subsequence also denoted by $\rho_{n}$ that converges strongly towards the function $\rho$ in $L^{2}\left(Q_{T}\right)$. Since $G$ is closed we deduce that $\rho \in G$. Finally, from (4.13) we deduce that $\theta \in \Lambda^{-1}(G)$. This completes the proof of Proposition 4.1.

\section{Acknowledgments}

This work was improved at the Universite de Versailles. The author is grateful to Professor J.-P. Puel for helpful suggestions.

\section{References}

[1] B. Ainseba, Exact and approximate controllability of the age and space population dynamics structured model, Journal of Mathematical Analysis and Applications 275 (2002), no. 2, 562-574.

[2] B. Ainseba and S. Anita, Internal exact controllability of the linear population dynamics with diffusion, Electronic Journal of Differential Equations 2004 (2004), no. 112, 1-11.

[3] B. Ainseba and M. Iannelli, Exact controllability of a nonlinear population-dynamics problem, Differential and Integral Equations 16 (2003), no. 11, 1369-1384.

[4] B. Ainseba and M. Langlais, Sur un problème de contrôle d'une population structurée en âge et en espace, Comptes Rendus de l'Académie des Sciences. Série I. Mathématique 323 (1996), no. 3, 269-274.

[5] C. Avramescu, A fixed point theorem for multivalued mappings, Electronic Journal of Qualitative Theory of Differential Equations 2004 (2004), no. 17, 1-10. 


\section{Controllability of a nonlinear population model}

[6] A. V. Fursikov and O. Yu. Imanuvilov, Controllability of Evolution Equations, Lecture Notes Series, vol. 34, Seoul National University Research Institute of Mathematics Global Analysis Research Center, Seoul, 1996.

[7] P. Martinez, J.-P. Raymond, and J. Vancostenoble, Regional null controllability of a linearized Crocco-type equation, SIAM Journal on Control and Optimization 42 (2003), no. 2, 709-728.

[8] O. Nakoulima, A. Omrane, and J. Velin, A nonlinear problem for age-structured population dynamics with spatial diffusion, Topological Methods in Nonlinear Analysis 17 (2001), no. 2, 307 319.

[9] J. P. Puel, Application of global Carleman inequalities to controllability and inverses problems, notes of courses, 2003.

[10] O. Traore and A. Ouedraogo, Sur un problème de dynamique des populations, IMHOTEP. Journal Africain de Máthematiques Pures et Appliquées 4 (2003), no. 1, 15-23.

Oumar Traore: Département de Mathématiques, Université de Ouagadougou, Ouagadougou 03, BP 7021, Burkina Faso

E-mail address: traore.oumar@univ-ouaga.bf 


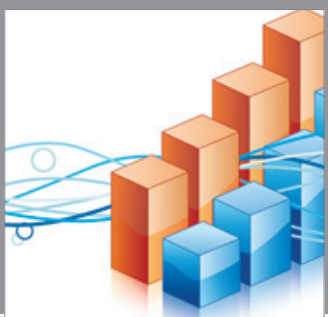

Advances in

Operations Research

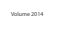

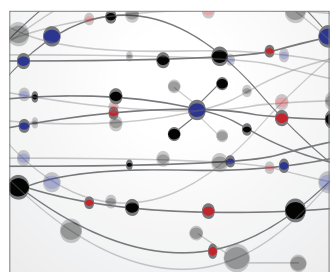

\section{The Scientific} World Journal
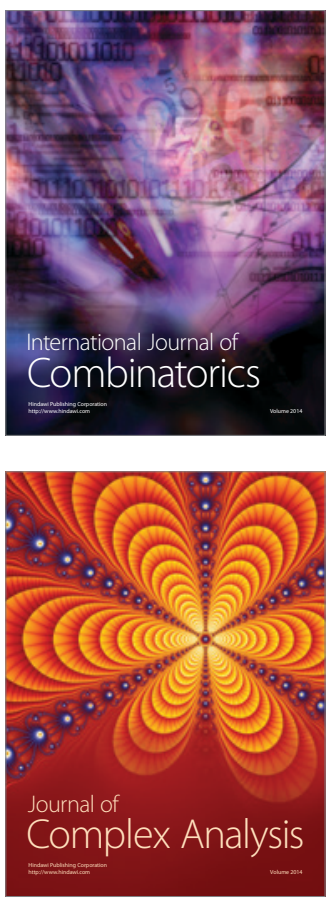

International Journal of

Mathematics and

Mathematical

Sciences
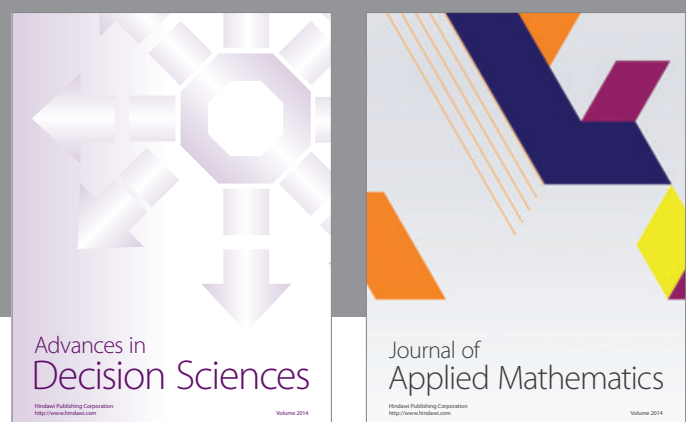

Journal of

Applied Mathematics
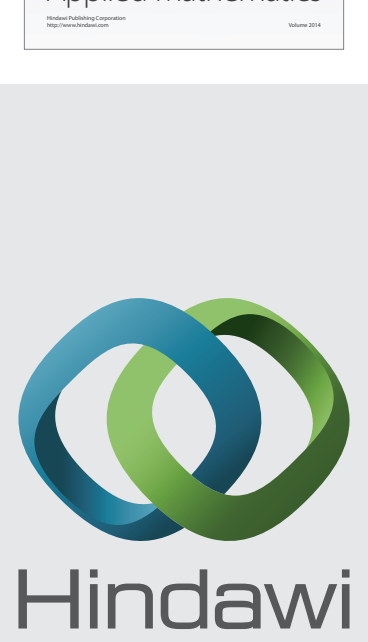

Submit your manuscripts at http://www.hindawi.com
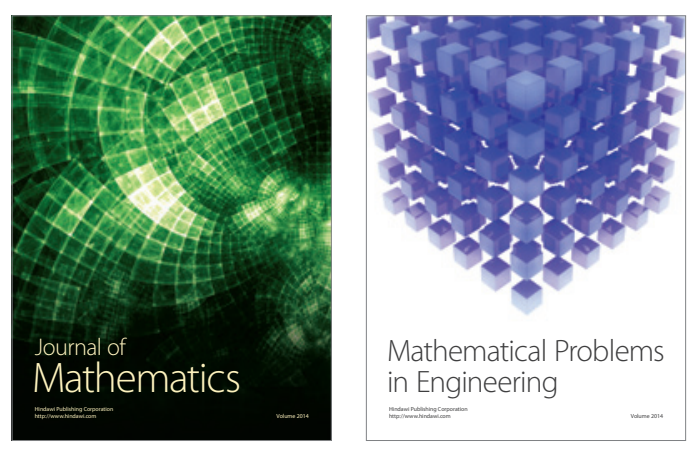

Mathematical Problems in Engineering
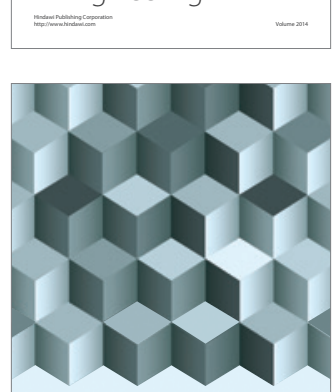

Journal of

Function Spaces
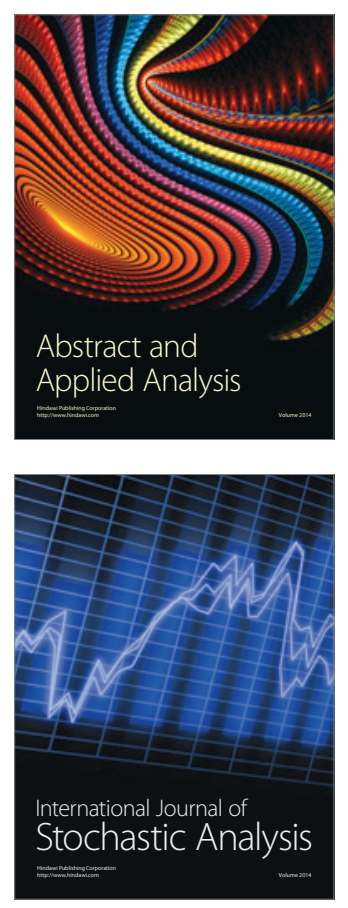

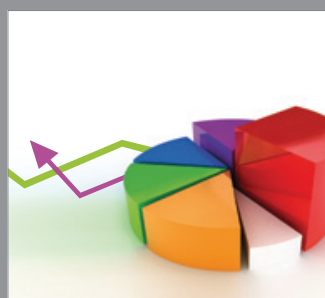

ournal of

Probability and Statistics

Promensencen
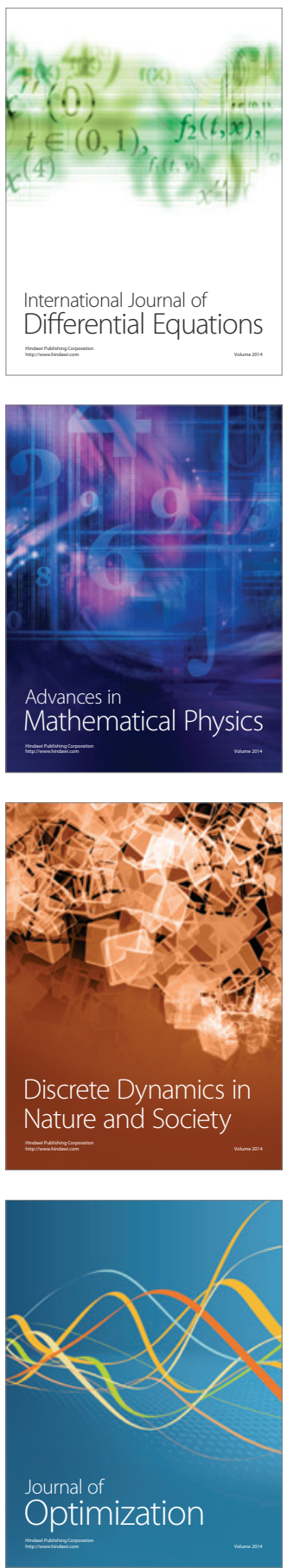